\title{
Effect of Fentanyl and Morphine on Gallbladder Dimensions in Newborns Admitted to the Neonatal Intensive Care Unit: A Randomized Double-Blinded Clinical Trial
}

\author{
Salma Naderi, ${ }^{1}$ Rakhshaneh Goodarzi, ${ }^{2}$ Gholam Reza Payrov Naziri, ${ }^{3}$ Ahmad Malek Mohammad, ${ }^{4}$ \\ Azita Kheiltash, , $^{*}$ and Ahmad Shafaeizadeh ${ }^{6}$ \\ ${ }^{1} \mathrm{MD}$, Department of Pediatric, Faculty of Medicine, Clinical Research Development Center of Children Hospital, Hormozgan University of Medical Sciences, Bandar Abbas, IR \\ Iran \\ ${ }^{2} \mathrm{MD}$, Clinical Research Development Center of Children Hospital, Hormozgan University of Medical Sciences, Bandar Abbas, IR Iran \\ ${ }^{3} \mathrm{MD}$, Pediatrician at Mashhad Petroleum Industry Health Organization \\ ${ }^{4} \mathrm{MD}$, Department of Radiology, Faculty of Medicine, Hormozgan University of Medical Sciences, Bandar Abbas, IR Iran \\ ${ }^{5} \mathrm{MD}, \mathrm{MPH}$, Department of Community Medicine, School of Medicine, Tehran University of Medical Sciences, Tehran, IR Iran \\ ${ }^{6}$ MD, MPH, Community Medicine Specialist, Saveh University of Medical Sciences, Amirkabir University of Technology \\ "Corresponding author: Azita Kheiltash, Building No.4, Faculty of Medicine, Poorsina St., Keshavarz Bul., And P.O.BOX: 14155-6447. Tel: +98-2164053216, Fax: +98-2188952357, \\ E-mail: kheiltash@tums.ac.ir
}

Received 2016 February 17; Revised 2016 June 15; Accepted 2016 November 26.

\begin{abstract}
Background: The effect of fentanyl and morphine, two most frequently used drugs for premedication in general anesthesia, on gallbladder of newborns, has not been sufficiently investigated. Intubation and mechanical ventilation in a neonatal respiratory distress syndrome are stressful procedures, in which these two drugs are used to reduce stress and pain. We studied the effect of these drugs on neonatal bile system during these procedures

Objectives: The present study aimed at determining the effect of fentanyl and morphine on gallbladder dimensions among newborns admitted to the neonatal intensive care unit (NICU).

Methods: Thirty-two newborns with the gestational age between 26 and 38 weeks, undergoing intubation and mechanical ventilation, were selected for this randomized, double-blinded, clinical trial study. They were randomly divided into two groups; group 1 received morphine and group 2 received fentanyl. Gallbladder measurements (width, depth, length and volume) were compared between the two groups by ultrasound evaluation. The data were collected between Nov 2012 and Oct 2013 at the NICU of the Children's hospital in Bandar Abbas, Iran.

Results: There were no significant differences between the two groups in the evaluated parameters when demographic variables were compared. We found statistically differences between width, depth and volume of the newborns' gallbladders. However, there was no significant differences with regard to gallbladder length $(\mathrm{P}=0.19)$.

Conclusions: Morphine caused gallbladder dilatation more than fentanyl. However, it should be considered that total parenteral nutrition (TPN) which is common in NICU leads to gallbladder dilatation. Therefore, it seems that fentanyl could be considered as a preferred short-term analgesic with less adverse effects than morphine. A larger study is needed to confirm these results.
\end{abstract}

Keywords: Fentanyl, Morphine, Gallbladder, Ultrasound, Neonate

\section{Background}

Respiratory distress syndrome (RDS) is the most common reason for the hospitalization of preterm and term newborns in the neonatal intensive care units (NICUs) (1). Neonatal intubation and mechanical ventilation are stressful procedures that require premedication. Premedication helps to improve biochemical and clinical conditions, and reduce stress, pain and adverse physiological responses. Pain and stress, induced during these procedures, may cause short-term and long-term side effects in newborns (2-4).

Drugs with maximal analgesic effect and minimal side effects are recommended as a premedication in the NICU, during neonatal intubation and mechanical ventilation (5, 6). Strong opioids such as morphine or fentanyl are frequently used in NICUs for reducing pain induced during these invasive procedures $(7,8)$. The best chosen opioid is the one with the strongest analgesic effect and minimal side effects $(9,10)$. It has been established that fentanyl is 50-100 times more potent than morphine in terms of pain reduction $(11,12)$.

There are very few clinical trial studies that have evaluated the effects of opioid drugs, such as fentanyl, on gallbladder dimensions and biliary tract dynamics, especially 
in newborns. In view of this, we conducted a randomized, double-blinded clinical trial to study the side effects of the most popular analgesic drugs (morphine and fentanyl) on gallbladder dimensions among newborns admitted to the NICUs for intubation and mechanical ventilation and also to compare the side effects of these two drugs. As far as we know, such a study has not been conducted in the NICU before.

\section{Objectives}

This study aimed at evaluating some gastrointestinal (GI) side effects of analgesic drugs (morphine and fentanyl) using ultrasound measurements of gallbladder and biliary tract dimensions and shape.

\section{Methods}

In this prospective, randomized, double-blinded clinical trial, 32 newborns with gestational age between 26 and 38 weeks undergoing intubation and mechanical ventilation (for at least 24 hours) were included. Parents of the participants were interviewed by a pediatrician on the necessity of using premedication for their newborns to reduce pain and stress and parental consent obtained. The research project was approved by the ethical committee and conducted under the supervision of Hormozgan University of Medical Sciences.

The participants were randomly divided into two groups (group 1 received morphine and group 2 received fentanyl). These two groups were further assigned to $<30$ weeks and $>30$ weeks subgroups, according to their gestational age. The data were gathered between Nov 2012 and Oct 2013 at the NICU of the Children's hospital in Bandar Abbas, Iran. This children's hospital with 130 beds is affiliated to Hormozgan University of Medical Sciences for education and training, and serves as a referral hospital of the region.

\subsection{Study Population}

This study population consisted of 32 newborns aged 26 - 38 weeks with similar demographic variables, who were admitted to the NICU of the Children's hospital in Bandar Abbas for mechanical ventilation, following the respiratory distress syndrome, bacterial pneumonia, viral pneumonia, and other respiratory problems for at least 24 hours during a period of 12 months. Forty-six patients were assessed. Among them, 5 could not meet the inclusion criteria and parents of 9 newborns opted to not participate in the study.
Patients were assessed for participation in the study based on the following inclusion and exclusion criteria:

Inclusion Criteria:

Newborns

- With a gestational age of 24 weeks or more,

- Admitted for mechanical ventilation at the NICU,

- Whose parents gave permission to the research team to get involved in this study (parental consent)

Exclusion Criteria:

Newborns with

- Asphyxia (possible hepatic or renal damage),

- APGAR scores below 5 at 5 th minute after birth,

- Direct hyperbilirubinemia,

- Neonatal hepatitis,

- Biliary system anomalies,

- Cardiovascular anomalies,

- Gastrointestinal obstructions.

Newborns that met the inclusion criteria were stratified randomly into two groups using the closed envelope's method. One of the NICU nurses who was not involved in taking the vital signs, physical examination, and registration of the newborns administered the allocated drugs. Group 1 received morphine prescribed with a loading dose of $100 \mathrm{mcg} / \mathrm{kg}$ in the first hour, and a maintenance dose of $12 \mathrm{mcg} / \mathrm{kg} /$ hour for the next 24 hours, injected intravenously. Group 2 received fentanyl. It was prescribed with a loading dose of $2 \mathrm{mcg}$, and with a maintenance dose of $0.25 \mathrm{mcg} / \mathrm{kg} /$ hour, intravenously injected slowly. These dose adjustments were being administered based on experience from previous studies and reference book resources $(10,13)$.

Ultrasonographic evaluation of the newborns was performed by a radiologist who had no prior information about the two groups. Ultrasonographic measurements of gallbladder dimensions (length, width, depth and volume) as well as the occurrence of hydrops of gall bladder were evaluated on the third and sixth days after drug administration. Our radiologist used a sonogragraphy transducer at $7.5 \mathrm{MHz}$ for all the measurements. All the newborns in this study were under total parenteral nutrition (TPN) before ultrasound evaluation.

All data were classified based on the newborns' age (day), weight (gram), gestational age (week), respiratory conditions and ultrasound results. We used SPSS 13 for the evaluation of the data. Because statistical distribution of the samples in both groups had a normal distribution pattern, a t test was used to compare the means of both groups. P value $<0.05$ was considered as statistically significant. 


\subsection{Trial Registerion Code}

Register: IRCT, ID: IRCT201105216532N1; URL: http://www.irct.ir/searchresult.php?id=6532\&number=1; available at: http://apps.who.int/trialsearch/Trial2.aspx? TrialID=IRCT201105216532N1.

\section{Results}

Thirty-two newborns undergoing mechanical ventilation at the NICU were randomly divided into two groups containing 16 newborns each. Morphine was prescribed and administered to newborns in group 1 and, fentanyl was prescribed for the newborns in group 2. Each group was randomly stratified into two subgroups based on gestational age; $>30$ weeks or $<30$ weeks. Each subgroup contained 8 newborns with gestational age either $>30$ weeks or $<30$ weeks (Table 1 ).

Table 1. Demographic Characteristics and Physiologic Variables of the Patients ${ }^{\mathrm{a}}$

\begin{tabular}{|lcc|}
\hline & Fentanyl Group & Morphine Group \\
\hline Sex & & \\
\multicolumn{1}{|c}{ Male } & $10(62.5)$ & $9(56.2)$ \\
\hline \multicolumn{1}{c}{ Female } & $6(37.5)$ & $7(43.8)$ \\
\hline Gestational age, $\mathbf{w}$ & $31.6 \pm 4.7$ & $31.0 \pm 4.2$ \\
\hline Birth weight, $\mathbf{g}$ & $916 \pm 1714$ & $790 \pm 1661$ \\
\hline Study duration, $\mathbf{d}$ & $1.1 \pm 5.8$ & $1.4 \pm 5.5$ \\
\hline Ventilator Usage Time, $\mathbf{d}$ & $1.1 \pm 5.8$ & $1.8 \pm 5.0$ \\
\hline Day of sonography & $0.9 \pm 3.9$ & $1.4 \pm 4.6$ \\
\hline Sum & 16 & 16 \\
\hline
\end{tabular}

${ }^{\mathrm{a}}$ Values are expressed as mean \pm SD or No. (\%).

Table 1 shows the results of comparison between the two groups in terms of their demographic characteristics. There were no significant differences between the two groups when demographic variables were compared.

We observed no significant differences between the two groups when we compared their total and direct serum bilirubin levels. A total serum bilirubin level in newborns who received fentanyl was between $2.1-12.9 \mathrm{mg} / \mathrm{dL}$ (average: $5.56 \mathrm{mg} / \mathrm{dL}$ ), whereas that of the morphine- administered group was between 2.3 - $8.50 \mathrm{mg} / \mathrm{dL}$ (average: $4.27 \mathrm{mg} / \mathrm{dL}$ ), $\mathrm{P}=0.22$. Similar results were observed in direct serum bilirubin levels among the newborns.

Preliminary diagnosis for these newborns at the time of admission to hospital were RDS in 24 (75\%), sepsis in 5 $(15 \%)$, and miscellaneous in 3 newborns.

Ultrasound evaluation of the newborns was performed by a single radiologist using the same device. It was performed between 3 to 6 days (average: 4.15 days) after the first opioid administration. Eighty-four percent of the newborns had ultrasound evaluation up to the 5th day after opioid administration. Table 2 shows the dimensions and volume (14) of gallbladder of the newborns in the two groups.

Table 2. Sonographic Measurements of Gallbladder (Length, Width, Depth and Volume) in the Fentanyl Group Compared to the Morphine Group

\begin{tabular}{lccc}
\hline & Fentanyl Group & Morphine Group & P Value \\
\hline $\begin{array}{l}\text { Gallbladder } \\
\text { length, mm }\end{array}$ & $22 \pm 5.12$ & $24.3 \pm 4.6$ & 0.19 \\
$\begin{array}{l}\text { Gallbladder width, } \\
\text { mm }\end{array}$ & $7.3 \pm 1.7$ & $11.2 \pm 2.4$ & $<0.0001$ \\
$\begin{array}{l}\text { Gallbladder Depth, } \\
\text { mm }\end{array}$ & $6.1 \pm 1.74$ & $9.4 \pm 2$ & $<0.0001$ \\
$\begin{array}{l}\text { Gallbladder } \\
\text { Volume, } \text { mm }^{3}\end{array}$ & $0.65 \pm 0.35$ & $2.04 \pm 1.08$ & $<0.0001$ \\
\hline
\end{tabular}

The results of gallbladder measurements showed that all parameters, except for the length of gallbladder, were higher in morphine-administered newborns than in the newborns who received fentanyl (Figure 1).

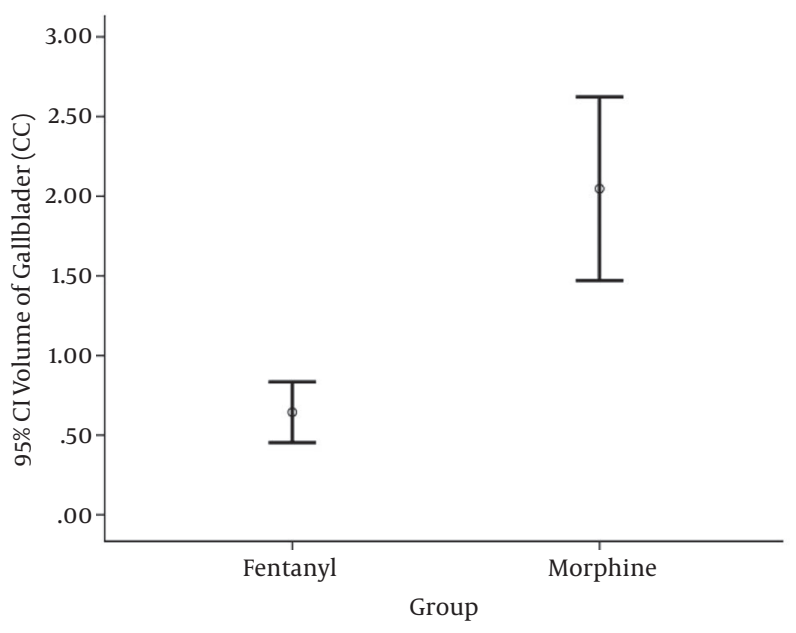

Figure 1. Gallbladder Volume of Morphine and Fentanyl Groups Showed a Significant Difference (t Test)

\section{Discussion}

Endotracheal intubation for mechanical ventilation of newborns triggers physiological and clinical responses against pain, which are followed by certain side effects. Choosing the best analgesic with maximum analgesic effect and minimum side effects is very important $(6,7)$.

Morphine and fentanyl are the two most commonly used opioids in NICUs. In this study, we tried to evaluate the 
side effects of these drugs using ultrasound measurements of gallbladder and biliary tract dimensions and shape.

A significant finding, which had not been reported previously was dilation of gallbladder which happened more remarkable in newborns who received morphine in comparison to newborns who got fentanyl. Some studies have shown that long-term prescription of fentanyl in adults may cause a decreased biliary flow, which in turn raises intrabiliary pressure in the common biliary duct (CBD) and sphincter of Oddi (14-16). However, we did not observe such changes in the CBD diameter by ultrasound evaluation in the two groups. This difference may be due to the fact that our study lacked a long-term follow-up. There are few studies that have investigated the effects of morphine on the biliary system. In one study, gallbladder stone formation in mothers addicted to morphine was reported (17). In a study conducted by Zsigmond et al., the injection of morphine significantly reduced the diameter of the bile duct (18). However, Viera et al., using ultrasonographic measurements, reported no significant changes in the diameter of the common bile duct after fentanyl injection (19).

Similar to our study, Schmidt et al. evaluated the effect of opioids on the biliary system of newborns (20). The results showed that the administration of fentanyl had no effects on the length, width and volume of the gallbladder in newborns. The sample size and fentanyl maintenance dose in the Schmidt's study were not significantly different compared with those in our study, but the average gestational age and birth weight of the newborns in his study were higher than ours. Also, in both studies the technique used in the ultrasound evaluation in both studies was the same. However, it should be noticed that total parenteral nutrition (TPN), which is common in the ICU, intensifies gallbladder dilatation (21). Therefore, we consider fentanyl a preferred short-term analgesic with less adverse effects than morphine to be used in NICU.

We compared changes in gallbladder dimensions in both morphine-and fentanyl-treated groups. To the best of our knowledge, no similar study has so far been conducted in this regard. However, some studies have reported that in adults morphine and fentanyl can raise the pressure inside the biliary duct and the sphincter of Oddi. Morphine can also decrease the diameter of the common bile duct but fentanyl has not been found to have the same effect. Considering the size and diameter of the common bile duct in newborns, especially premature neonates, this effect may cause biliary stasis and dilatation, and in a long-term period, may cause hydrops of the gallbladder. There were no records of direct hyperbilirubinemia in any of the cases evaluated in this study.

\subsection{Limitations}

There were some ethical issues regarding the administration of analgesics to neonates who underwent mechanical ventilation. These issues prevented us from comparing the two groups of newborns who received morphine and fentanyl with a group of newborns who received placebo as premedication. Other limitations of the study were the small sample size, which may be attributed to the limited number of beds in the NICU, and the limited period of study time. Insufficient ultrasound devices in our NICU was another difficulty we faced in our study. In certain cases, we needed multiple or same time sonographic evaluation. Considering the limited period of time in which this study was conducted (average of 7 days for each patient), we were not able to monitor the values of gallbladder dilatation for a longer time in the newborns who received maintenance doses of morphine. Furthermore, we were not able to evaluate gallbladder dimension or occurrence of hydrops after the study period.

\subsection{Recommendations}

Studies should be conducted on a larger population to assess other GI signs and side effects and to confirm these results. Moreover, better evaluation methods should be used to establish the association between the side effects of these drugs and gestational age, birth weight, having TPN versus enteral nutrition and prescription duration. Assessment of other complications including necrotizing enterocolitis (NEC), early and late onset sepsis (EOS and LOS), and the final outcome in these neonates could also help to make better decision about the drugs (22). Sonographic evaluation of gallbladder before the administration of opioids, during their administration and even after discontinuation of these drugs will be crucial in defining precisely their effect on the biliary system.

\subsection{Conclusions}

As an adverse effect in newborns that receive morphine or fentanyl as a premedication, morphine causes gallbladder dilatation more than fentanyl. However, it should be noticed that TPN, which is commonly used the ICU, intensifies gallbladder dilatation. Therefore, fentanyl could be considered as a preferred short-term analgesic drug with less adverse effects than morphine in NICU. A larger study is needed to assess other GI signs and side effects and to confirm these results.

\section{Acknowledgments}

We would like to thank all the colleagues who helped us throughout this project, in particular the neonatal and 
intensive care unit nurses of the Children's hospital in Bandar Abbas.

\section{Footnote}

Funding/Support: This study was financially supported by the department of pediatrics, faculty of medicine, Hormozgan University of Medical Sciences, Bandar Abbas, Iran.

\section{References}

1. Johnston CC, Collinge JM, Henderson SJ, Anand KJ. A cross-sectional survey of pain and pharmacological analgesia in Canadian neonatal intensive care units. Clin J Pain. 1997;13(4):308-12. [PubMed: 9430811].

2. Anand KJ. Clinical importance of pain and stress in preterm neonates. Biol Neonate. 1998;73(1):1-9. [PubMed: 9458936].

3. Barker DP, Rutter N. Stress, severity of illness, and outcome in ventilated preterm infants. Arch Dis Child Fetal Neonatal Ed. 1996;75(3):F18790. [PubMed: 8976685].

4. Evans DJ, MacGregor RJ, Dean HG, Levene MI. Neonatal catecholamine levels and neurodevelopmental outcome: a cohort study. Arch Dis Child Fetal Neonatal Ed. 2001;84(1):F49-52. [PubMed: 11124926].

5. Hall RW, Boyle E, Young T. Do ventilated neonates require pain management?. Semin Perinatol. 2007;31(5):289-97. doi: 10.1053/j.semperi.2007.07.002. [PubMed: 17905183].

6. Ranger M, Johnston CC, Anand KJS. Current controversies regarding pain assessment in neonates. Seminars in perinatology. Saunders; 2007. pp. 283-8.

7. Tibboel D, Anand KJ, van den Anker JN. The pharmacological treatment of neonatal pain. Semin Fetal Neonatal Med. 2005;10(2):195-205. doi: 10.1016/j.siny.2004.11.002. [PubMed: 15701584].

8. Anand KJ, Hall RW, Desai N, Shephard B, Bergqvist LL, Young TE, et al. Effects of morphine analgesia in ventilated preterm neonates: primary outcomes from the NEOPAIN randomised trial Lancet. 2004;363(9422):1673-82. doi: 10.1016/S0140-6736(04)16251-X. [PubMed: 15158628].

9. A joint statement of the Fetus and Newborn Committee . Prevention and management of pain and stress in the neonate. Paediatr Child Health. 2000;5(1):31-47. [PubMed: 20107594]
10. Saarenmaa E, Huttunen P, Leppaluoto J, Meretoja O, Fellman V. Advantages of fentanyl over morphine in analgesia for ventilated newborn infants after birth: A randomized trial. J Pediatr. 1999;134(2):144-50. [PubMed: 9931520].

11. Carbajal R, Rousset A, Danan C, Coquery S, Nolent P, Ducrocq $S$, et al. Epidemiology and treatment of painful procedures in neonates in intensive care units. JAMA. 2008;300(1):60-70. doi: 10.1001/jama.300.1.60. [PubMed: 18594041].

12. Carbajal R, Lenclen R, Jugie M, Paupe A, Barton BA, Anand KJ. Morphine does not provide adequate analgesia for acute procedural pain among preterm neonates. Pediatrics. 2005;115(6):1494-500. doi: 10.1542/peds.2004-1425. [PubMed: 15930209].

13. Fanaroff AAM, Fanaroff RA, Martin RJ, Klaus MHF, Avroy A. Neonatalperinatal medicine: diseases of the fetus and infant(Expert Consult Online and Print). Elsevier Health Sciences; 2002.

14. Hynynen MJ, Turunen MT, Korttila KT. Effects of alfentanil and fentanyl on common bile duct pressure. Anesth Analg. 1986;65(4):370-2. [PubMed: 3082247]

15. McCammon RL, Stoelting RK, Madura JA. Effects of butorphanol, nalbuphine, and fentanyl on intrabiliary tract dynamics. Anesth Analg. 1984;63(2):139-42. [PubMed: 6691579].

16. Tremblay PR, Poncelet P, Dinh DK. [Fentanyl and biliary tract pressure]. Can Anaesth Soc J. 1973;20(6):747-53. [PubMed: 4769608].

17. Figueroa-Colon R, Tolaymat N, Kao SC. Gallbladder sludge and lithiasis in an infant born to a morphine user mother.JPediatr Gastroenterol Nutr. 1990;10(2):234-8. [PubMed: 2303975].

18. Zsigmond EK, Vieira ZE, Duarte B, Renigers SA, Hirota K. Double-blind placebo-controlled ultrasonographic confirmation of constriction of the common bile duct by morphine. Int J Clin Pharmacol Ther Toxicol. 1993;31(10):506-9. [PubMed: 8262689].

19. Vieira ZE, Zsigmond EK, Duarate B, Renigers SA, Hirota K. Evaluation of fentanyl and sufentanil on the diameter of the common bile duct by ultrasonography in man: a double blind, placebo controlled study. Int J Clin Pharmacol Ther. 1994;32(6):274-7. [PubMed: 7921524].

20. Schmidt B, Roth B, Stutzer H, Benz-Bohm G. Prospective sonographic evaluation of fentanyl side effects on the neonatal gallbladder. Eur J Clin Pharmacol. 2006;62(10):823-7. doi: 10.1007/s00228-006-0170-7. [PubMed: 16896787].

21. Schmidt B, Roth B, Stutzer H, Benz-Bohm G. Prospective volumetric analysis of gallbladders in critically ill newborns: the impact of nutrition. Neonatology. 2007;92(3):201-4. doi:10.1159/000102058. [PubMed: 17476120].

22. Afjeh SA, Sabzehei MK, Fahimzad AR, Shiva F, Shamshiri AR, Esmaili F. Antibiotic Therapy for Very Low Birth Weigh Newborns in NICU. Iran J Pediatr. 2016;26(2):e2612. doi: 10.5812/ijp.2612. [PubMed: 27307961]. 